\title{
Theory of Fuzzy Sets: Construction of Membership Surfaces of Normal Fuzzy Vectors
}

\author{
Hemanta K. Baruah \\ Vice-Chancellor, Bodoland University, Kokrajhar, India \\ hemanta_bh@yahoo.com
}

\begin{abstract}
Fuzzy vectors have been studied almost since the beginning of the theory of fuzzy sets. However, just as a fuzzy number has to be defined with reference to a membership function, every fuzzy vector too has to be defined with reference to a membership surface. Nothing however is available in the literature about the membership surface when a fuzzy vector is defined. In this article, using the Randomness-Fuzziness Consistency Principle, it would be shown how to construct the membership surface of a normal fuzzy vector.
\end{abstract}

Keywords: Fuzzy membership function, fuzzy membership surface, fuzzy vector

\section{Introduction}

Every fuzzy real number has to be defined with reference to a membership function. In the same way, every fuzzy vector too has to be defined with reference to what can be called a membership surface. It is true that vector addition and vector subtraction can be explained with reference to the membership functions associated to the real numbers defining the vectors concerned. However, in the literature, there is no mention of anything like a membership surface. In this article, it would be shown how the membership surface of a fuzzy vector can be constructed.

In the Dubois-Prade representation of a normal fuzzy number, the membership function $\mu(x)$ of a fuzzy number $[a, b, c]$ is given by $\mu(x)$

$$
\begin{aligned}
& =L(x), a \leq x \leq b, \\
& =R(x), b \leq x \leq c, \\
& =1, \text { otherwise, }
\end{aligned}
$$

where $L(x)$ is a continuous non-decreasing function and $R(x)$ is continuous nonincreasing function where

$$
\begin{gathered}
L(a)=R(c)=0, \\
L(b)=R(b)=1 .
\end{gathered}
$$

Using the idea of superimposition of sets put forward by the present author [1], it was shown [2-6] that the Dubois-Prade left reference function $L(x)$ is a distribution function of a random variable, and that the Dubois-Prade right reference function $R(x)$ is a complementary distribution function of another random variable. In this article, using this idea it would be shown how to construct the membership surface of a normal fuzzy vector.

In Section-2, we would discuss the classical concept of Order Statistics which is available in standard text books of Statistics. This concept may however be new to many researchers working on fuzzy mathematics. We shall therefore elaborate the essential features concerned in this Section. The Glivenko-Cantelli theorem on empirical probability distribution functions is sometimes considered as the backbone of distribution theory in Statistics. This theorem would be deduced in this Section. Thereafter, in 
Section-3, we would discuss the Mathematics of Partial Presence defined by the present author [6]. It would be shown how the concept of order statistics helps us to define partial presence of an element in an interval. How to represent a normal fuzzy vector based on the mathematics of partial presence would be discussed in Section-4. In Section-5, we would discuss how to construct the membership surface of a normal fuzzy vector. Finally, discussions on the findings would be made in Section-6.

\section{Order Statistics}

Let $X_{1}, X_{2}, \ldots, X_{\mathrm{n}}$ denote a random sample from a population with continuous cumulative distribution function $F_{X}$. The distribution is continuous, and so the probability of any two or more of these random variables assuming equal magnitudes is zero. Therefore, there would exist a unique ordered arrangement of the sample values. At this point and hereafter, we would like the readers to note that $X_{1}, X_{2}, \ldots, X_{\mathrm{n}}$ may be ordered already in the sense that there is no probability law of errors governing their occurrences, and even then what now follows would be mathematically true (see $e . g .$, [7-11]).

Let $X_{(1)}$ denote the smallest, $X_{(2)}$ denote the second smallest, and finally $X_{(\mathrm{n})}$ denote the largest of $X_{1}, X_{2}, \ldots, X_{\mathrm{n}}$. Then

$$
X_{(1)}<X_{(2)}<\ldots<X_{(\mathrm{n})}
$$

would be the original sample after arrangement in the ascending order of magnitude. $X_{(\mathrm{r})}$ here is known as the $r$-th order statistics. As $F_{X}$ is continuous, the random variable

$$
y=F_{X}(x)
$$

is uniformly distributed in the interval $(0,1)$. Indeed, because

$$
0 \leq F_{X}(x) \leq 1
$$

for all $x$, we have

$$
\begin{aligned}
F_{Y}(y) & =0 \text { if } y \leq 0, \\
& =1 \text { if } y \geq 1 .
\end{aligned}
$$

For $0<y<1$, writing $F_{X}(u)=y$, where $u$ is the largest number satisfying this, it can be seen that $F_{X}(x) \leq y$ is necessary as well as sufficient for $X \leq u$. Accordingly,

$$
F_{Y}(y)=F_{X}(u)=y,
$$

thus leading us to the uniform law of randomness.

Accordingly, if $X_{1}, X_{2}, \ldots, X_{\mathrm{n}}$ are random with the continuous distribution function $F_{X}$, then

$$
F_{X}\left(X_{1}\right), F_{X}\left(X_{2}\right), \ldots, F_{X}\left(X_{\mathrm{n}}\right)
$$

are uniformly random. In the same way, for

also

$$
X_{(1)}<X_{(2)}<\ldots<X_{(\mathrm{n})}
$$

would be uniformly random.

$$
F_{X}\left(X_{(1)}\right), F_{X}\left(X_{(2)}\right), \ldots, F_{X}\left(X_{(\mathrm{n})}\right)
$$

An empirical distribution function $S_{n}(x)$ for a sample of size $n$ is defined as the proportion of values that do not exceed $x$. Accordingly, if $X_{(1)}, X_{(2)}, \ldots, X_{(\mathrm{n})}$ denote the order statistics of a random sample, its empirical distribution function would be given by $S_{n}(x)$

$$
\begin{aligned}
& =0, \text { if } x<X_{(1),} \\
& =k / n, \text { if } X_{(k)} \leq x<X_{(\mathrm{k}+1)}, k=1,2, \ldots,(n-1), \\
& =1, \text { if } x \geq X_{(n)} .
\end{aligned}
$$


$X$ here being random, so would be $S_{n}(X)$. It can be seen that we would have

$$
\operatorname{Prob}\left[S_{n}(x)=k / n\right]={ }^{n} C_{k}\left[F_{X}(x)\right]^{k}\left[1-F_{X}(x)\right]^{n-k}
$$

for $k=0,1, \ldots, n$. Hence the mathematical expectation of $S_{n}(x)$ would be

$$
E\left[S_{n}(x)\right]=F_{X}(x) .
$$

Therefore, $S_{n}(x)$ converges uniformly to $F_{X}(x)$ almost surely.

This leads to the Glivenko - Cantelli theorem: The limiting value of the supremum of the difference between $\mathrm{S}_{n}(x)$ and $F_{X}(x)$, as $n$ becomes infinitely large, converges to zero almost surely. In other words, a function

$$
\begin{aligned}
& S_{n}(x)=0, \text { if } x<X_{(1),} \\
& \quad=k / n \text {, if } X_{(k)} \leq x<X_{(\mathrm{k}+1)}, k=1,2, \ldots,(n-1), \\
& \quad=1, \text { if } x \geq X_{(n)}
\end{aligned}
$$

as $n$ tends to infinity, becomes a distribution function.

\section{The Mathematics of Partial Presence}

Defined by the present author [1], the operation of set superimposition is expressed as follows. If the set A is superimposed over the set $\mathrm{B}$, we get

$$
A(S) B=(A-B) \cup(A \cap B)^{(2)} \cup(B-A)
$$

where $S$ represents the operation of superimposition, and $(A \cap B){ }^{(2)}$ represents the elements of $(A \cap B)$ occurring twice, when $(A \cap B)$ is not void. This operation was introduced keeping view that fact that when two line segments $\mathrm{A}$ and $\mathrm{B}$ of unequal lengths are overdrawn one over the other, this is what happens. It can be seen that for two intervals $A=\left[a_{1}, b_{1}\right]$ and $B=\left[a_{2}, b_{2}\right]$, we get

$$
\begin{gathered}
\quad\left[a_{1}, b_{1}\right](S)\left[a_{2}, b_{2}\right] \\
=\left[a_{1}, a_{2}\right] \cup\left[a_{2}, b_{1}\right]^{(2)} \cup\left[b_{1}, b_{2}\right], \text { if } a_{1}<a_{2}<b_{1}<b_{2}, \\
=\left[a_{1}, a_{2}\right] \cup\left[a_{2}, b_{2}\right]^{(2)} \cup\left[b_{2}, b_{1}\right], \text { if } a_{1}<a_{2}<b_{2}<b_{1}, \\
=\left[a_{2}, a_{1}\right] \cup\left[a_{1}, b_{1}\right]^{(2)} \cup\left[b_{1}, b_{2}\right], \text { if } a_{2}<a_{1}<b_{1}<b_{2}, \\
=\left[a_{2}, a_{1}\right] \cup\left[a_{1}, b_{2}\right]^{(2)} \cup\left[b_{2}, b_{1}\right], \text { if } a_{2}<a_{1}<b_{2}<b_{1},
\end{gathered}
$$

where
i) $a_{1}<a_{2}<b_{1}<b_{2}$,
ii) $a_{1}<a_{2}<b_{2}<b_{1}$,
iii) $a_{2}<a_{1}<b_{1}<b_{2}$
iv) $a_{2}<a_{1}<b_{2}<b_{1}$

are the four possibilities in this case. Here we have assumed that $\left[a_{1}, b_{1}\right] \cap\left[a_{2}, b_{2}\right]$ is not void, or in other words

$$
\max \left(a_{i}\right) \leq \min \left(b_{i}\right), i=1,2 .
$$

Indeed

where

$$
\left[a_{1}, b_{1}\right](S)\left[a_{2}, b_{2}\right]=\left[a_{(1)}, a_{(2)}\right] \cup\left[a_{(2)}, b_{(1)}\right]^{(2)} \cup\left[b_{(1)}, b_{(2)}\right]
$$

i) $\mathrm{a}_{(1)}=\min \left(\mathrm{a}_{1}, \mathrm{a}_{2}\right)$,

ii) $\mathrm{a}_{(2)}=\max \left(\mathrm{a}_{1}, \mathrm{a}_{2}\right)$,

iii) $b_{(1)}=\min \left(b_{1}, b_{2}\right)$, and

iv) $b_{(2)}=\max \left(b_{1}, b_{2}\right)$. 
This step of expressing the result in terms of ordered values is an important one. We would soon find how this conversion helps us to arrive at our final results.

For three intervals $A=\left[a_{1}, b_{1}\right], B=\left[a_{2}, b_{2}\right]$ and $C=\left[a_{3}, b_{3}\right]$, such that $\left[a_{1}, b_{1}\right] \cap\left[a_{2}, b_{2}\right]$ $\cap\left[\mathrm{a}_{3}, \mathrm{~b}_{3}\right]$ is not void, or

it can be seen that

$$
\max \left(a_{i}\right) \leq \min \left(b_{i}\right), i=1,2,3,
$$

$$
\begin{aligned}
& {\left[a_{1}, b_{1}\right](S)\left[a_{2}, b_{2}\right](S)\left[a_{3}, b_{3}\right]} \\
& =\left[\mathrm{a}_{(1)}, \mathrm{a}_{(2)}\right] \cup\left[\mathrm{a}_{(2)}, \mathrm{a}_{(3)}\right]^{(2)} \cup\left[\mathrm{a}_{(3)}, \mathrm{b}_{(1)}\right]^{(3)} \cup\left[\mathrm{b}_{(1)}, \mathrm{b}_{(2)}\right]^{(2)} \cup\left[\mathrm{b}_{(2)}, \mathrm{b}_{(3)}\right] \text {, }
\end{aligned}
$$

where $a_{(1)}, a_{(2)}, a_{(3)}$ are values of $a_{1}, a_{2}, a_{3}$ arranged in increasing order of magnitude, and $b_{(1)}, b_{(2)}, b_{(3)}$ are values of $b_{1}, b_{2}, b_{3}$ arranged in increasing order of magnitude, where for example $\left[\mathrm{a}_{(2)}, \mathrm{a}_{(3)}{ }^{(2)}\right.$ are elements of $\left[\mathrm{a}_{(2)}, \mathrm{a}_{(3)}\right]$ represented twice.

Using induction it can be seen that for $n$ intervals

$$
\left[a_{1}, b_{1}\right],\left[a_{2}, b_{2}\right], \ldots,\left[a_{n}, b_{n}\right],
$$

subject to

$$
\left[a_{1}, b_{1}\right] \cap\left[a_{2}, b_{2}\right] \cap \ldots \cap\left[a_{n-1}, b_{n-1}\right] \cap\left[a_{n}, b_{n}\right]
$$

is not void, we would have (n!) ${ }^{2}$ different cases that can in short be written as

$$
\begin{gathered}
{\left[a_{1}, b_{1}\right](S)\left[a_{2}, b_{2}\right](S) \ldots(S)\left[a_{n-1}, b_{n-1}\right](S)\left[a_{n}, b_{n}\right]} \\
=\left[a_{(1)}, a_{(2)}\right] \cup\left[a_{(2)}, a_{(3)}\right]^{(2)} \cup \ldots \cup\left[a_{(n-1)}, a_{(n)}\right]^{(n-1)} \\
\cup\left[a_{(n)}, b_{(1)}\right]^{(n)} \cup\left[b_{(1)}, b_{(2)}{ }^{(n-1)} \cup \ldots\right. \\
\cup\left[b_{(n-2)}, b_{(n-1)}\right]^{(2)} \cup\left[b_{(n-1)}, b_{(n)}\right]
\end{gathered}
$$

where $a_{(1)}, a_{(2)}, \ldots, a_{(n)}$ are values of $a_{1}, a_{2}, \ldots, a_{n}$ arranged in increasing order of magnitude, and $b_{(1)}, b_{(2)}, \ldots, b_{(n)}$ also are values of $b_{1}, b_{2}, \ldots, b_{n}$ arranged in increasing order of magnitude, and for example $\left[\mathrm{a}_{(\mathrm{n}-1)}, \mathrm{a}_{(\mathrm{n})}\right]^{(\mathrm{n}-1)}$ are elements of $\left[\mathrm{a}_{(\mathrm{n}-1)}, \mathrm{a}_{(\mathrm{n})}\right]$ represented (n-1) times. Oder statistical matters would now automatically enter into our discussions on application of set superimposition.

We now proceed towards an application of the operation of set superimposition. We refer to the example of two line segments $\mathrm{A}$ and $\mathrm{B}$ drawn one over the other again. Double representation creates a doubly dark situation in the common portion. In the same way, when an opaque object is placed in front of a source of light, we can see umbra and penumbra on a screen placed nearby. Now on a line drawn over the shadow, it can be seen that the line segment over the umbra would be doubly dark in comparison to the line segments over the penumbra. If the level of darkness on the doubly dark portion is taken to be unity, that on the less dark portions it would have to be fractional, $1 / 2$ say. These are two practical examples of partial presence of an element in a set.

In other words, if $\left[a_{1}, b_{1}\right]^{(1 / 2)}$ and $\left[a_{2}, b_{2}\right]^{(1 / 2)}$ represent two intervals both with level of presence $1 / 2$ for every real number in the intervals, superimposition of the two intervals $\left[a_{1}\right.$, $\left.\mathrm{b}_{1}\right]^{(1 / 2)}$ and $\left[\mathrm{a}_{2}, \mathrm{~b}_{2}\right]^{(1 / 2)}$ would give rise to

$$
\begin{gathered}
{\left[\mathrm{a}_{1}, \mathrm{~b}_{1}\right]^{(1 / 2)}(\mathrm{S})\left[\mathrm{a}_{2}, \mathrm{~b}_{2}\right]^{(1 / 2)}} \\
=\left[\mathrm{a}_{(1)}, \mathrm{a}_{(2)}\right]^{(1 / 2)} \cup\left[\mathrm{a}_{(2)}, \mathrm{b}_{(1)}\right]^{(1)} \cup\left[\mathrm{b}_{(1)}, \mathrm{b}_{(2)}\right]^{(1 / 2)} .
\end{gathered}
$$

It is similar to placing one translucent paper over another of equal opacity $1 / 2$ to get the opacity doubled as a result in the common portion.

In the same way, if $\left[a_{1}, b_{1}\right]^{(1 / 3)},\left[a_{2}, b_{2}\right]^{(1 / 3)}$ and $\left[a_{3}, b_{3}\right]^{(1 / 3)}$ represent three intervals all with level of presence equal to $1 / 3$ for every real number in the intervals, superimposition of $\left[a_{1}, b_{1}\right]^{(1 / 3)},\left[a_{2}, b_{2}\right]^{(1 / 3)}$ and $\left[a_{3}, b_{3}\right]^{(1 / 3}$ would give rise to 


$$
\begin{gathered}
{\left[\mathrm{a}_{1}, \mathrm{~b}_{1}\right]^{(1 / 3)}(\mathrm{S})\left[\mathrm{a}_{2}, \mathrm{~b}_{2}\right]^{(1 / 3)}(\mathrm{S})\left[\mathrm{a}_{3}, \mathrm{~b}_{3}\right]^{(1 / 3)}} \\
=\left[\mathrm{a}_{(1)}, \mathrm{a}_{(2)}\right]^{(1 / 3)} \cup\left[\mathrm{a}_{(2)}, \mathrm{a}_{(3)}\right]^{(2 / 3)} \cup\left[\mathrm{a}_{(3)}, \mathrm{b}_{(1)}\right]^{(1)} \cup\left[\mathrm{b}_{(1)}, \mathrm{b}_{(2)}\right]^{(2 / 3)} \cup\left[\mathrm{b}_{(2)}, \mathrm{b}_{(3)}\right]^{(1 / 3)}
\end{gathered}
$$

So for $n$ intervals $\left[a_{1}, b_{1}\right]^{(1 / n)},\left[a_{2}, b_{2}\right]^{(1 / n)}, \ldots,\left[a_{n}, b_{n}\right]^{(1 / n)}$ all with level of presence equal to $1 / \mathrm{n}$ for every real number in the intervals, we shall have

$$
\begin{gathered}
{\left[\mathrm{a}_{1}, \mathrm{~b}_{1}\right]^{(1 / \mathrm{n})}(\mathrm{S})\left[\mathrm{a}_{2}, \mathrm{~b}_{2}\right]^{(1 / \mathrm{n})}(\mathrm{S}) \ldots(\mathrm{S})\left[\mathrm{a}_{\mathrm{n}}, \mathrm{b}_{\mathrm{n}}\right]^{(1 / \mathrm{n})}} \\
=\left[\mathrm{a}_{(1)}, \mathrm{a}_{(2)}\right]^{(1 / \mathrm{n})} \cup\left[\mathrm{a}_{(2)}, \mathrm{a}_{(3)}\right]^{(2 / \mathrm{n})} \cup \ldots \cup\left[\mathrm{a}_{(\mathrm{n}-1)}, \mathrm{a}_{(\mathrm{n})}\right]^{(\mathrm{n}-1) / \mathrm{n})} \\
\cup\left[\mathrm{a}_{(\mathrm{n})}, \mathrm{b}_{(1)}\right]^{(1)} \cup\left[\mathrm{b}_{(1)}, \mathrm{b}_{(2)}\right]^{(\mathrm{n}-1) / \mathrm{n})} \cup \ldots \cup\left[\mathrm{b}_{(\mathrm{n}-2)}, \mathrm{b}_{(\mathrm{n}-1)}\right]^{(2 / \mathrm{n})} \cup\left[\mathrm{b}_{(\mathrm{n}-1)}, \mathrm{b}_{(\mathrm{n})}\right]^{(1 / \mathrm{n})},
\end{gathered}
$$

where, for example, $\left[\mathrm{b}_{(1)}, \mathrm{b}_{(2)}\right]^{((\mathrm{n}-1) / \mathrm{n})}$ represents the interval $\left[\mathrm{b}_{(1)}, \mathrm{b}_{(2)}\right]$ with level of presence $((n-1) / n)$ for every real number in the interval, $\mathrm{a}_{(1)}, \mathrm{a}_{(2)}, \ldots \ldots \ldots, \mathrm{a}_{(\mathrm{n})}$ being values of $a_{1}, a_{2}, \ldots \ldots \ldots, a_{n}$ arranged in increasing order of magnitude, and $b_{(1)}, b_{(2)}$, , $b$ (n) being values of $b_{1}, b_{2}, \ldots \ldots \ldots, b_{n}$ arranged in increasing order of magnitude.

Consider two probability spaces $\left(\Omega_{1}, A_{1}, \Pi_{1}\right)$ and $\left(\Omega_{2}, A_{2}, \Pi_{2}\right)$ where $\Omega_{1}$ and $\Omega_{2}$ are intervals $[\alpha, \beta]$ and $[\beta, \gamma]$ respectively on the real line. Let $a_{1}, a_{2}, \ldots, a_{\mathrm{n}}$ and $b_{1}, b_{2}, \ldots, b_{\mathrm{n}}$ be realizations in $[\alpha, \beta]$ and $[\beta, \gamma]$ respectively. Recall now the intervals $\left[a_{1}, b_{1}\right]^{(1 / n)},\left[a_{2}\right.$, $\left.b_{2}\right]^{(1 / \mathrm{n})}, \ldots,\left[a_{\mathrm{n}}, b_{\mathrm{n}}\right]^{(1 / \mathrm{n})}$, all with constant level of presence $1 / n$ for every real number in the intervals. The levels of presence are

$$
1 / n, 2 / n, \ldots,(n-1) / n, 1,(n-1) / n, \ldots, 2 / n, 1 / n \text {. }
$$

Observe that the values of levels of presence can be considered in two parts,

and

$$
(0,1 / n, 2 / n, \ldots,(n-1) / n, 1)
$$

$$
(1,(n-1) / n, \ldots, 2 / n, 1 / n, 0) \text {. }
$$

They can define an empirical distribution and a complementary empirical distribution on $a_{1}, a_{2}, \ldots, a_{\mathrm{n}}$ and $b_{1}, b_{2}, \ldots, b_{\mathrm{n}}$ respectively. It can be seen that if we define

$$
\begin{aligned}
\Pi_{1}(x) & =0, \text { if } x<a_{(1)}, \\
& =(r-1) / n, \text { if } a_{(\mathrm{r}-1)} \leq x \leq a_{(\mathrm{r})}, r=2,3, \ldots, n, \\
& =1, \text { if } x \geq a_{(\mathrm{n})}, \\
\Pi_{2}(\mathrm{x}) & =1, \text { if } x<b_{(1)}, \\
& =1-(r-1) / n, \text { if } b_{(\mathrm{r}-1)} \leq x \leq b_{(\mathrm{r})}, r=2,3, \ldots, n, \\
& =0, \text { if } x \geq a_{(\mathrm{n})},
\end{aligned}
$$

as $n$ tends to infinity

$$
\begin{gathered}
\Pi_{1}(\mathrm{x}) \rightarrow \Psi_{1}(x), \alpha \leq x \leq \beta, \\
\Pi_{2}(\mathrm{x}) \rightarrow\left(1-\Psi_{2}(x)\right), \beta \leq x \leq \gamma
\end{gathered}
$$

where $\Psi_{1}(x), \alpha \leq x \leq \beta$, and $\left(1-\Psi_{2}(x)\right), \beta \leq x \leq \gamma$, are the underlying theoretical distribution functions.

This shows that there is equivalence between the definitions of the Dubois - Prade left reference function of a normal fuzzy number and the probability distribution function of a random variable, and there is equivalence between the definitions of the Dubois - Prade right reference function of a normal fuzzy number and the complementary distribution function of a random variable. For every distribution function, there must be a density function.

It can be observed that we have arrived at the Dubois-Prade definition of normal fuzzy numbers, and therefore at the Zadehian definition of normal fuzzy numbers, without actually using the definition of fuzziness. We have started with our definition of partial 
presence and have established that the two definitions lead to the classical definition of fuzziness. Accordingly, it is clear that we have neither modified nor generalized the existing definition of fuzziness.

Thus two densities $\mathrm{d} \Psi_{1}(x) / \mathrm{d} x$ and $\mathrm{d}\left(1-\Psi_{2}(x)\right) / \mathrm{d} x$ in the intervals $[\alpha, \beta]$ and $[\beta, \gamma]$ respectively can define a normal fuzzy number $N=[\alpha, \beta, \gamma]$ with membership function

$$
\begin{gathered}
\mu_{N}(x)=\Psi_{1}(x), \text { if } \alpha \leq x \leq \beta, \\
=\Psi_{2}(x), \text { if } \beta \leq x \leq \gamma, \\
=0, \text { otherwise. }
\end{gathered}
$$

For a normal fuzzy number of the type $N=[\alpha, \beta, \gamma]$ with membership function

with

$$
\begin{aligned}
& \mu_{N}(x)=\Psi_{1}(x), \text { if } \alpha \leq x \leq \beta, \\
& =\Psi_{2}(x), \text { if } \beta \leq x \leq \gamma, \text { and } \\
& =0, \text { otherwise, }
\end{aligned}
$$

$$
\begin{aligned}
& \Psi_{1}(\alpha)=\Psi_{2}(\gamma)=0, \\
& \Psi_{1}(\beta)=\Psi_{2}(\beta)=1,
\end{aligned}
$$

the partial presence of a value $x$ of the variable $X$ in the interval $[\alpha, \gamma]$ is expressible as

with

$$
\mu_{N}(x)=\theta \Psi_{1}(x)+(1-\theta) \Psi_{2}(x),
$$

$$
\begin{aligned}
\theta=1, \text { if } \alpha & \leq x \leq \beta, \text { and } \\
& =0, \text { if } \beta \leq x \leq \gamma .
\end{aligned}
$$

We can therefore conclude that given two intervals $[\alpha, \beta]$ and $[\beta, \gamma]$, two probability measures defined on these two intervals can define a normal fuzzy number $[\alpha, \beta, \gamma]$. The distribution function in the interval $[\alpha, \beta]$ and the complementary distribution function in the interval $[\beta, \gamma]$ can together form the membership function of the fuzzy number. It may be observed that applying the operation of superimposition of intervals with levels of presence of real numbers, we could arrive at the definition of a normal fuzzy number. The Zadehian definition of fuzzy numbers has been there since 1965. However, the actual mathematical meaning of normal fuzziness can be explained using our mathematics of partial presence only.

\section{Representation of a Normal Fuzzy Vector}

For a normal fuzzy real number $[a, b, c]$, the membership function $\mu(x)$ for $a \leq x \leq c$ is a curve. For a normal fuzzy vector $\{[a, b, c],[d, e, f]\}$, where $[a, b, c]$ and $[d, e, f]$ are two normal fuzzy real numbers, the membership function has to be a surface. Here $[a, b, c]$ is a normal fuzzy number defined on the horizontal axis and $[d, e, f]$ is a normal fuzzy number defined on the vertical axis. The question is: how to describe the membership surface.

Let

$$
\begin{gathered}
\mu_{X}(x)=L_{X}(x), a \leq x \leq b, \\
=R_{X}(x), b \leq x \leq c, \\
=1, \text { otherwise, and } \\
\mu_{Y}(y)=L_{Y}(y), d \leq y \leq e, \\
=R_{Y}(y), e \leq y \leq f, \\
=1, \text { otherwise, }
\end{gathered}
$$


be the two membership functions of the normal fuzzy real numbers $[a, b, c]$ and $[d, e, f]$. Here,

$$
\begin{aligned}
& L_{X}(x)=\operatorname{Prob}[a \leq X \leq x], a \leq x \leq b, \\
& L_{Y}(y)=\operatorname{Prob}[d \leq Y \leq y], d \leq y \leq e, \\
& R_{X}(x)=1-\operatorname{Prob}[b \leq X \leq x], b \leq x \leq c, \\
& R_{Y}(y)=1-\operatorname{Prob}[e \leq Y \leq y], e \leq y \leq f
\end{aligned}
$$

for two random variables $X$ and $Y$ ([2], [3], [4], [5], [6]).

Now, just as a fuzzy normal real number $[a, b, c]$ defined on the horizontal axis is described in two parts, the intervals $[a, b]$ and $[b, c]$, a normal fuzzy vector of the type $\{[a, b, c],[d, e, f]\}$ with the fuzzy coordinate $[a, b, c]$ defined with reference to the horizontal axis and the fuzzy coordinate $[d, e, f]$ defined with reference to the vertical axis, can be described in four parts, four rectangles to be precise. Indeed, the real number $b$ was expressed as a normal fuzzy real number $[a, b, c]$, while the real number $e$ was expressed as a normal fuzzy real number $[d, e, f]$. Accordingly, the vector $(b, e)$ on the $X Y$ plane can be expressed on the $X Y$ plane as a normal fuzzy vector $\{[a, b, c],[d, e, f]\}$.

Consider the four rectangles defined by the vertices:

$$
\begin{array}{lc}
\text { i) } & \mathrm{A}(b, e), \mathrm{B}(a, e), \mathrm{C}(a, d), \mathrm{D}(b, d), \\
\text { ii) } & \mathrm{A}(b, e), \mathrm{E}(b, f), \mathrm{F}(a, f), \mathrm{B}(a, e), \\
\text { iii) } & \mathrm{A}(b, e), \mathrm{G}(c, e), \mathrm{H}(c, f), \mathrm{E}(b, f), \\
\text { iv) } & \mathrm{A}(b, e), \mathrm{D}(b, d), \mathrm{I}(c, d), \mathrm{H}(c, e) .
\end{array}
$$

These four rectangles together form the rectangle with extreme points $\mathrm{C}(a, d), \mathrm{I}(c, d)$, $\mathrm{H}(c, f)$ and $\mathrm{F}(a, f)$. The rectangle CIHF defines the normal fuzzy vector $\{[a, b, c],[d, e$, $f]$ \}. In the next Section, it would be shown how to construct the membership surface of this normal fuzzy vector.

\section{Construction of Membership Surface}

Just as superimposition of $n$ intervals $\left[\mathrm{a}_{1}, \mathrm{~b}_{1}\right]^{(1 / \mathrm{n})},\left[\mathrm{a}_{2}, \mathrm{~b}_{2}\right]^{(1 / \mathrm{n})}, \ldots,\left[\mathrm{a}_{\mathrm{n}}, \mathrm{b}_{\mathrm{n}}\right]^{(1 / \mathrm{n})}$ all with level of presence equal to $1 / \mathrm{n}$ for every real number in the intervals has led us to define a normal fuzzy number, to get a normal fuzzy vector we could have gone forward to superimpose $n$ rectangles with random extreme points. Indeed, this would have been the most natural extension of our operation of set superimposition. However, this procedure would be too complicated to deal with mathematically. Further, for 3 and higher dimensional vectors, this procedure would not be further extendable. Therefore, we have taken the following simpler path to arrive at the solution.

Consider the rectangle $\mathrm{ABCD}$ mentioned in the earlier Section. $\mathrm{AB}$ here is the interval in which the membership function is

$$
L_{X}(x)=\operatorname{Prob}[a \leq X \leq x], a \leq x \leq b,
$$

while $\mathrm{AD}$ is the interval in which the membership function is

$$
L_{Y}(y)=\operatorname{Prob}[d \leq Y \leq y], d \leq y \leq e .
$$

Therefore the joint probability that $a \leq X \leq x$ and $d \leq Y \leq y$, when $a \leq x \leq b, d \leq y \leq e$, would be given by the product of the two probabilities as the random variables $X$ and $Y$ are independent of each other. This is where lies the physical significance of the definition our membership surface of a normal fuzzy vector.

In other words,

$$
\begin{gathered}
\operatorname{Prob}[a \leq X \leq x, d \leq Y \leq y], \\
=\{\operatorname{Prob}[a \leq X \leq x] . \operatorname{Prob}[d \leq Y \leq y]\}
\end{gathered}
$$


for $a \leq x \leq b, d \leq y \leq e$. Hence, the membership of $(x, y)$ for $a \leq x \leq b$ and $d \leq y \leq e$ would have to be the product

$$
\{\text { Prob }[a \leq X \leq x] . \text { Prob }[d \leq Y \leq y]\}
$$

which is nothing but $L_{X}(x) L_{Y}(y)$ for $a \leq x \leq b, d \leq y \leq e$.

In the other three rectangles too, the same logic would be applicable. Finally, the function defining the membership surface would be

$$
\begin{gathered}
\mu_{X, Y}(x, y)=L_{X}(x) L_{Y}(y), a \leq x \leq b, d \leq y \leq e \\
=L_{X}(x) R_{Y}(y), a \leq x \leq b, e \leq y \leq f \\
=R_{X}(x) L_{Y}(y), b \leq x \leq c, d \leq y \leq e \\
=R_{X}(x) R_{Y}(y), b \leq x \leq c, e \leq y \leq f
\end{gathered}
$$

We have so far discussed how to construct the fuzzy membership surface of a 2dimensional vector. The fuzzy membership hyper-surfaces of vectors in higher dimensions can be constructed generalizing this idea. For a 2-dimensional fuzzy vector, the membership surface can be expressed as shown above, in the form of four products defined on four rectangles. For 3-dimensional fuzzy vector, the membership hyper-surface can be expressed in the form of eight products defined on eight cuboids.

The properties of the left reference function and the right reference function are already known as they were expressed in the Dubois-Prade definition of normal fuzzy numbers. Therefore the properties of products such as $L_{X}(x) L_{Y}(y), a \leq x \leq b, d \leq y \leq e$, would be in the lines of the standard properties of the Dubois-Prade reference functions.

Differentiation of the left reference function which is a probability distribution function, would give us the probability density function $[2,6]$ of a random variable. Similarly, differentiation of the right reference function which is a complementary probability distribution function, would lead to the probability density function of another random variable. The same explanations would be valid for products such as $L_{X}(x) L_{Y}(y)$, $a \leq x \leq b, d \leq y \leq e$.

\section{Conclusions and Discussions}

Dubois and Prade rightly understood that the membership function $\mu(x)$ of a fuzzy number $[a, b, c]$ has to be described as two different functions $L(x)$ and $R(x)$ in two intervals $[a, b]$ and $[b, c]$. They further observed that $L(x)$ is a continuous non-decreasing function and $R(x)$ is continuous non-increasing function where

$$
\begin{aligned}
& L(a)=R(c)=0, \\
& L(b)=R(b)=1 .
\end{aligned}
$$

However, they have not mentioned how to estimate these two functions with reference to possible construction of a fuzzy number $[a, b, c]$. Had they tried to see wherefrom these two functions appear, the history of fuzzy mathematics would perhaps have been very different.

The present author has observed that the mathematics of partial presence leads to fuzzy numbers and that $L(x)$ is nothing but a distribution function and that $R(x)$ is nothing but a complementary distribution function. As soon as that is accepted as true, the physical significance of the products:

(i) $\quad L_{X}(x) L_{Y}(y)$ for $a \leq x \leq b, d \leq y \leq e$,

(ii) $L_{X}(x) R_{Y}(y)$ for $a \leq x \leq b, e \leq y \leq f$,

(iii) $\quad R_{X}(x) L_{Y}(y)$ for $b \leq x \leq c, d \leq y \leq e$,

(iv) $\quad R_{X}(x) R_{Y}(y)$ for $b \leq x \leq c, e \leq y \leq f$, 
can be explained mathematically. Multiplying a left reference function $L(x)$ and a right reference function $R(y)$ would otherwise have no physical significance, and this might be the reason why no author has yet discussed anything about how to construct the membership surface of a normal fuzzy vector.

Ever since the theory of fuzzy sets was introduced by Zadeh [12] exactly half a century ago, thousands of articles appeared worldwide, modifying and generalizing his epoch making idea. However, how to construct the membership function of a normal fuzzy number strictly mathematically was never discussed. Therefore, the problem of constructing the membership surface of a normal fuzzy vector mathematically was never studied. In this article, it has been shown that construction of the membership surface of a normal fuzzy vector is a simple matter if one proceeds to do so using the RandomnessFuzziness Consistency Principle put forward by the present author [2].

In his Probability-Possibility Consistency Principle, Zadeh tried to establish some kind of consistency between a probability distribution that he tried to impose on the same interval on which a law of fuzziness was already defined. With reference to a normal fuzzy number $[a, b, c]$, a normal law of fuzziness in the interval $[a, c]$ can be explained with the help of two distinct probability laws, one in the interval $[a, b]$ and the other in the interval $[b, c]$; one single law of probability in the interval $[a, c]$ is not sufficient to explain a normal fuzzy number $[a, b, c]$. The present author has been trying to express this view since the nineties of the last century [1-6]. Indeed, if a variable is fuzzy, then it anyway is a meaningless exercise to try to transform it to a random variable. Similarly, if a variable is random, it is not clear why at all it should be related to the concept of fuzziness.

Zadeh's idea of finding a link between a probability distribution on the same interval on which a normal fuzzy number was already defined was equivalent to establishing a probability density function from the membership function of the normal fuzzy number defined on the interval. This has finally led others to define what is currently known as fuzzy measure. However, according to the Randomness-Fuzziness Consistency Principle defined by the present author [2], two probability measures are necessary and sufficient to describe a normal fuzzy number. Therefore we do not need to define a fuzzy measure that is not a measure in the classical sense. Thousands of articles have meanwhile been published on the fuzzy measure. It is now next to impossible to convince the fuzzy mathematics fraternity that a change of view is needed in this context. The researchers who have been working towards generalizing the concept of the fuzzy measure would like to continue their works in the accepted lines, instead of accepting the present author's views that two probability measures as defined in the classical theory of measure are enough to define every normal law of fuzziness.

The Random-Fuzziness Consistency Principle due to the present author was used to do fuzzy arithmetic without using the standard method of $\alpha$-cuts $[13,14]$. Results returned by our method were precisely the same as what were found by using the method of $\alpha$-cuts. In the method of $\alpha$-cuts, a line parallel to the horizontal axis is drawn, which cuts the membership function at two points. Our method returned the same results simply because the line parallel to the horizontal axis would cut at the same two points even if the membership function is defined in two distinct parts as in the Dubois-Prade representation. However, our method was based on our Randomness-Fuzziness Consistency Principle that states that the Dubois-Prade left reference function is a probability distribution function and the Dubois-Prade right reference function is a complementary probability distribution function. We would like to mention at this point that in this article, to define the membership surface of a normal fuzzy vector we have used the same principle that was used to do fuzzy arithmetic without using the standard method of $\alpha$-cuts. 
It is expected that there may be objections even in our current attempt to show how to construct the membership surface of a normal fuzzy vector. However, whatever has been put forward in this article is strictly mathematical; nothing heuristic has been included here.

\section{References}

[1] Hemanta K. Baruah, Set Superimposition and Its Application to the Theory of Fuzzy Sets, Journal of the Assam Science Society, Vol. 40, No. 1 \& 2, 1999, 25 - 31.

[2] Hemanta K. Baruah, The Randomness-Fuzziness Consistency Principle, International Journal of Energy, Information and Communications, Vol. 1, Issue 1, 2010, 37 - 48.

[3] Hemanta K. Baruah, In Search of the Root of Fuzziness: The Measure Theoretic Meaning of Partial Presence, Annals of Fuzzy Mathematics and Informatics, Vol. 2, No. 1, 2011, 57 - 68.

[4] Hemanta K. Baruah, The Theory of Fuzzy Sets: Beliefs and Realities, International Journal of Energy Information and Communications, Vol. 2, Issue 2, 2011, 1-22.

[5] Hemanta K. Baruah, Construction of the Membership Function of a Fuzzy Number, ICIC Express Letters, Vol. 5, Issue 2, 2011, 545-549.

[6] Hemanta K. Baruah, Construction of Normal Fuzzy Numbers Using the Mathematics of Partial Presence, Journal of Modern Mathematics Frontier, Vol. 1, No. 1, 2012, 9 - 15.

[7] M. Loeve, Probability Theory, Springer Verlag, New York, 1977.

[8] Jean Dickinson Gibbons and Subhabrata Chakraborti; Nonparametric Statistical Inference, Third Edition, Marcel Dekker, Inc., New York, 1992.

[9] M. Fisz; Theory of Probability and Mathematical Statistics, John Wiley \& Sons, New York, 1963.

[10] Robert B. Ash, and Catherine A. Doleans-Dade; Probability and Measure Theory, Academic Press, San Diego, 2000.

[11] V. K. Rohatgi and A. K. E. Saleh, An Introduction to Probability and Statistics, Second Edition, Wiley Series in Probability and Statistics, John Wiley \& Sons (Asia) Pte Ltd., Singapore, 2001.

[12] L. Zadeh, Fuzzy Sets, Information and Control, Vol. 8, 1965, 338 - 353.

[13] Rituparna Chutia, Supahi Mahanta and Hemanta K. Baruah, An Alternative Method of Finding the Membership of a Fuzzy Number, International Journal of Latest Trends in Computing, Vol. 1, Issue 2, 2010, 69-72.

[14] Supahi Mahanta, Rituparna Chutia and Hemanta K. Baruah, Fuzzy Arithmetic Without Using the method of $\alpha$-cuts, International Journal of Latest Trends in Computing, Vol. 1, Issue 2, 2010, 73-80.

\section{Author}

Hemanta Kumar Baruah is the Vice-Chancellor of Bodoland University, Kokrajhar, Assam, India, since September, 2013. After completing MSc in Statistics from Gauhati University in 1975, he obtained his $\mathrm{PhD}$ in Mathematics from Indian Institute of Technology, Kharagpur, in 1980. He joined the Imphal Campus of Jawaharlal Nehru University, New Delhi, as an Assistant Professor of Mathematics in September, 1979, and shifted to the Department of Statistics, Gauhati University, as Lecturer in March, 1981. In March, 1985, he became a Reader in Statistics, and was promoted to the position of Professor in June, 1995. He is currently on lien from Gauhati University. His research interests are in mathematics of fuzziness, graph theory, clustering algorithms and pattern recognition. 